\title{
EXPLOSIVE OUTFLOWS FROM FORMING MASSIVE STARS
}

\author{
J. Bally ${ }^{1}$, A. Ginsburg ${ }^{2}$ and M.M. Kasliwal ${ }^{3}$
}

\begin{abstract}
AO imaging of the near IR [Fe II] and $\mathrm{H}_{2}$ lines and ALMA $\mathrm{CO} \mathrm{J}=2-1$ data confirms the explosive nature of the BN/KL outflow in Orion. N-body interactions in compact groups may be responsible for the production of powerful, explosive protostellar outflows and luminous infrared flares. The Orion event may have been triggered by a protostellar merger. First results of a search for Orion-like events in 200 nearby galaxies with the SPitzer InfraRed Intensive Transients Survey (SPIRITS) are briefly discussed.
\end{abstract}

\section{Introduction}

The BN/KL region behind the Orion Nebula, located at a distance of $\sim 414 \mathrm{pc}$, contains a wide opening-angle, arcminute-scale outflow emerging from the OMC1 cloud core. It is traced by broad $\left(>100 \mathrm{~km} \mathrm{~s}^{-1}\right)$ emission lines at millimeter and sub-millimeter wavelengths, high-velocity maser emission, and bright shockexcited "fingers" of $\mathrm{H}_{2}$ and "fingertips" of $1.64 \mu \mathrm{m}$ [Fe II] emission (Bally et al. 2011, 2015). The BN/KL outflow contains hundreds of bow shocks (Fig. 1) some with proper motions of over $300 \mathrm{~km} \mathrm{~s}^{-1}$ and dynamic ages of 500 to 1,000 years.

The outflow contains at least $8 M_{\odot}$ of molecular gas with a median velocity of about $20 \mathrm{~km} \mathrm{~s}^{-1}$, and has a momentum and kinetic energy of at least $160 M_{\odot} \mathrm{km} \mathrm{s}^{-1}$ and $0.4-4 \times 10^{47} \mathrm{ergs}$, respectively (Snell 1984). Zapata et al. (2009) found that the CO flow consists of numerous bullets originating several arcseconds north of the OMC1 core with a dynamic age of $\sim 500$ years and noted that its explosive nature is different from accretion-disk powered jets and collimated bipolar protostellar outflows. These parameters imply a momentum injection rate of $\dot{P} \sim 0.3 M_{\odot} \mathrm{km} \mathrm{s}^{-1}$ year $^{-1}$.

Radio-frequency astrometry shows that the radio-emitting stars in OMC1, the Becklin-Neugebauer (BN) object and radio source I have space velocities of

\footnotetext{
${ }^{1}$ University of Colorado, Boulder, 80389, USA; e-mail: john.bally@colorado.edu

${ }^{2}$ ESO Headquarters, Garching bei Munchen, Germany

${ }^{3}$ Division of Physics, Mathematics, and Astronomy, California Institute of Technology, Pasadena, CA 91125, USA
} 


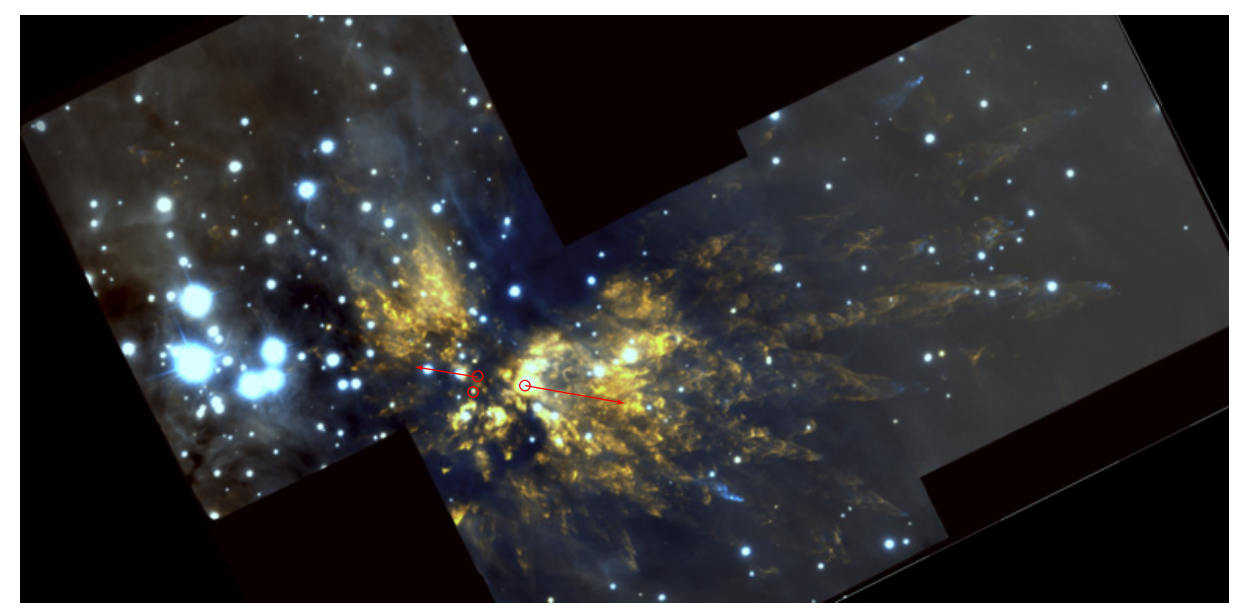

Fig. 1. The OMC1 BN/KL outflow in $\mathrm{H}_{2}$ (orange) and [Fe II] (blue) with locations of the BN object, source $\mathrm{I}$, and $\mathrm{n}$ indicated by red circles. The red vectors show the $21.3 \mathrm{~km} \mathrm{~s}^{-1}$ proper motions of BN (right) and the $14.3 \mathrm{~km} \mathrm{~s}^{-1}$ proper motion of source I (left) measured by Gomez (2008) and Goddi (2011). The lengths of the vectors are equal to the motion in 2,000 years. (e.g., $10^{\prime \prime}$ corresponds to a motion of $\sim 10 \mathrm{~km} \mathrm{~s}^{-1}$ ). Image is in Galactic coordinates.

21 and $14 \mathrm{~km} \mathrm{~s}^{-1}$ in opposite directions and away from a region less than $500 \mathrm{AU}$ in diameter from which they were ejected $\sim 500$ years ago (Gomez et al. 2008). The motions of BN and source I have been confirmed by many independent measurements (e.g., Goddi et al. 2011). The BN object, a 12-15 $M_{\odot}$ YSO has a radial velocity of $\mathrm{V}_{L S R}=+21 \mathrm{~km} \mathrm{~s}^{-1}$, receding with $\sim 11 \mathrm{~km} \mathrm{~s}^{-1}$ with respect to the OMC1 core (Scoville et al. 1983). Masers originating form radio source I indicate that it is approaching with a radial velocity of about $4 \mathrm{~km} \mathrm{~s}^{-1}$ with respect to OMC1. The ratio of the space motions of BN and source I away from the ejection center implies that source I has a mass $\sim 1.5$ times that of BN. The kinetic energy in the outflow and stellar motions today is $\sim 10^{48} \mathrm{ergs}$.

Bally and Zinnecker (2005) proposed that the OMC1 BN/KL outflow was triggered by the dynamic decay of a non-hierarchical system of massive stars which formed either a compact, AU scale binary, or led to a stellar merger. The interaction ejected the binary or merger remnant (suspected to be radio source I) and the BN object. Such explosive outflows may be associated with the ejection of runaway stars, the production of IR-flares with luminosities between novae and supernovae, and have profound feedback impact on their parent molecular clouds. In this model, the disruption and ejection of circumstellar disks and envelopes produced the BN/KL outflow. The momentum and kinetic energy of the outflow and ejected stars came from the release of gravitational binding energy of a compact binary or merger formed by the dynamic interaction of three of more stars (e.g., Reipurth \& Mikkola 2012). 


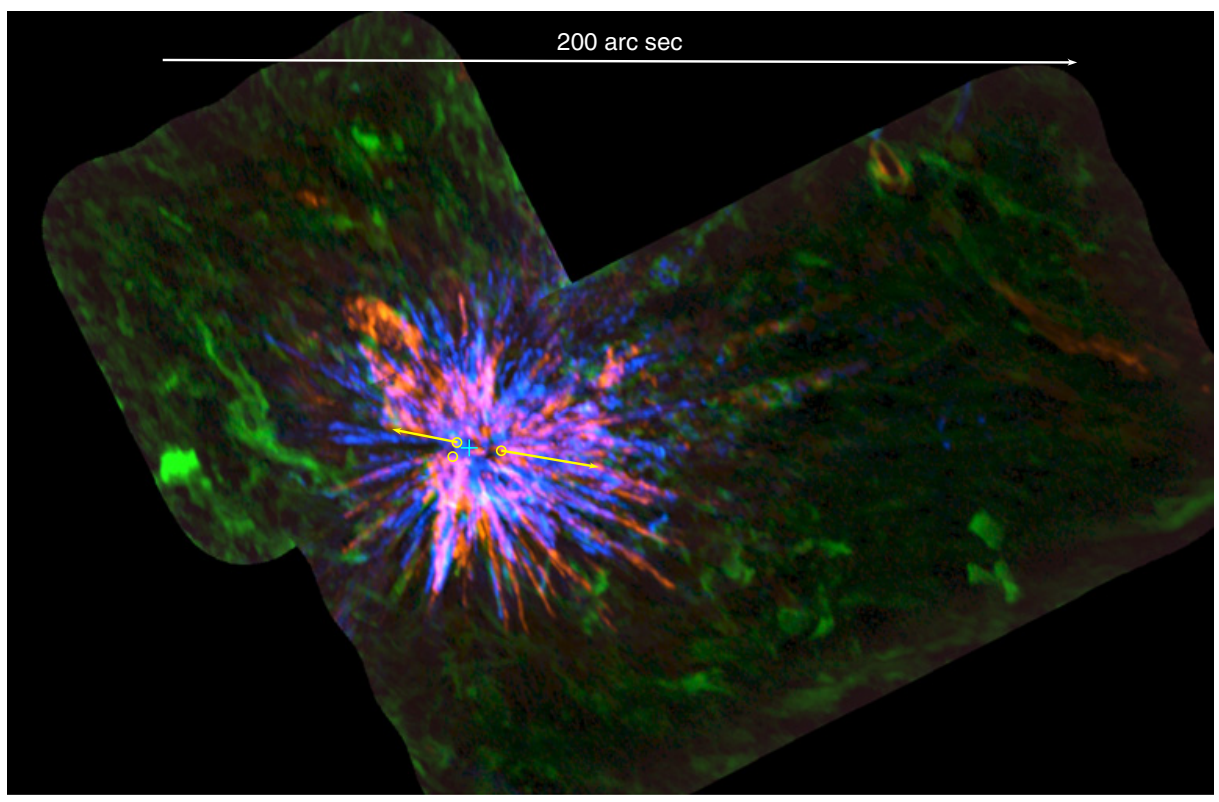

Fig. 2. An ALMA image showing the $230 \mathrm{GHz}$ CO J $=2-1$ emission from the BN/KL outflow with $1^{\prime \prime}(\sim 450 \mathrm{AU})$ resolution. Blueshifted emission is shown in blue, redshifted emission in red, and the emission within $\pm 10 \mathrm{~km} \mathrm{~s}^{-1}$ of the rest velocity of the OMC1 core is shown in green. Only the small scale structure in the cloud is shown by the green channel because of the spatial filtering effect of the ALMA interferometer. The yellow arrows show the motions of the BN object and source I as in Figure 1.

Figure 2 shows $\mathrm{CO}$ emission from the BN/KL outflow with $1^{\prime \prime}$ resolution (ALMA Cycle 2 program 2013.1.00546.S). The outflow is nearly isotropic with over 100 chains of arcsecond-wide CO streamers exhibiting linear velocity gradients with $V \propto D$, where $D$ is the distance form the suspected explosion center. Radial velocities extend to over $\pm 120 \mathrm{~km} \mathrm{~s}^{-1}$. These gradients also indicate a dynamic age of $\sim 500$ years. The image shows that the explosion originated between the current location of source I and BN (cyan plus-sign in Fig. 2).

Radio source I has not yet been directly detected at any infrared wavelength. However, it illuminates a $2 \mu \mathrm{m}$ reflection nebula about $10^{\prime \prime}$ to the east. The spectrum of this nebula indicates that source I has an effective photospheric temperature of $T_{\text {eff }} \approx 4,000 \mathrm{~K}$ similar to a $\mathrm{K}$ or $\mathrm{M}$ supergiant (Morino et al. 1998; Testi et al. 2010). Setting the accretion luminosity equal to the photospheric luminosity for an accretion rate $\dot{M} \sim 10^{-3} M_{\odot}$ year $^{-1}$ onto a star with mass $M_{*} \sim$ $10 M_{\odot}$, and solving for the radius gives $R_{*} \approx\left(G M_{*} \dot{M} / 4 \pi \sigma T_{\text {eff }}^{4}\right)^{1 / 3}$ $\sim 0.5 \mathrm{AU}$. The accretion luminosity at this radius is $L \sim 2.7 \times 10^{3} L_{\odot}$. The actual luminosity of source I is likely to be between $10^{4}$ and $10^{5} L_{\odot}$, which would result in a radius of 1.4 to 2.6 AU. Hosokawa \& Omukai (2009) present stellar structure 
models of massive $\left(\sim 10 M_{\odot}\right)$ stars accreting at high rates and show that they develop bloated photospheres and cool photospheric temperatures. For accretion rates $\dot{M}>10^{-3} M_{\odot} \mathrm{yr}^{-1}$, their models indicate that photospheric radii exceed $1 \mathrm{AU}$.

If the $\mathrm{BN} / \mathrm{KL}$ event was powered by the release of gravitational potential energy by the formation of a binary consisting of two $\sim 10 M_{\odot}$ stars, their separation would have to be a few AU. If one of the stars had an AU-scale photosphere, a star passing at this distance would have entered the massive stars's outer layers, possibly triggering a stellar merger. If the stellar cores merge, the mass of the cannibalized object has to be only $M>10^{48} R_{c} / G M_{*} \sim 0.04 M_{\odot}$ for a core radius $R_{c}=10^{11} \mathrm{~cm}$. Thus a merger with a low-mass companion can supply the observed energy. The merger-produced explosion would interact with the surrounding cloud core, resulting in mass-sorting of the ejecta which upon emergence from the core could produce the observed $\mathrm{CO}$ fingers, $\mathrm{H}_{2}$ wakes, and [Fe II] bullets.

The Spitzer SPIRITS program has detected dozens of luminous infrared flares in nearby galaxies. Most have no visual or near-IR counterparts, implying $T_{\text {eff }}<$ $10^{3} \mathrm{~K}$. A major spiral arm of the galaxy M83 hosted SPIRITS 14ajc which appeared on archival images in 2012, but was not recognized until 2014. Its absolute infrared magnitude at 3.6 and $4.5 \mu \mathrm{m}$ is -11 to -12 . A deep Keck spectrum revealed five lines in the $\mathrm{K}$-band ro-vibrational spectrum of $\mathrm{H}_{2}$ with intensity ratios similar to Orion but with $\mathrm{H}_{2}$ luminosity $>100 L_{\odot}$. No continuum was detected at any wavelength below $2.4 \mu \mathrm{m}$. N-body interactions in non-hierarchical multiples and in dense clusters may lead to the formation of binaries by capture and protostellar mergers. Such events may be responsible for a new class of luminous IR transients, the ejection of massive runaway stars, and be a major source of feedback in massive star forming regions.

\section{References}

Bally, J., \& Zinnecker, H., 2005, AJ, 129, 2281

Bally, J., Cunningham, N.J., Moeckel, N., et al., 2011, ApJ, 727, 113

Bally, J., Ginsburg, A., Silvia, D., \& Youngblood, A., 2015, A\&A, 579, A130

Goddi, C., Humphreys, E.M.L., Greenhill, L.J., et al., D., 2011, ApJ, 728, 15

Gomez, L., Rodŕguez, L.F., Loinard, L., et al., 2008, ApJ, 685, 333

Hosokawa, T., \& Omukai, K., 2009, ApJ, 691, 823

Morino, J.I., Yamashita, T., Hasegawa, T., \& Nakano, T., 1998, Nature, 393, 340

Reipurth, B., \& Mikkola, S., 2012, Nature, 492, 221

Scoville, N., Kleinmann, S.G., Hall, D.N.B., et al., 1983, ApJ, 275, 201

Snell, R.L., Scoville, N.Z., Sanders, D.B., et al., 1984, ApJ, 284, 176

Testi, L., Tan, J.C., \& Palla, F., 2010, A\&A, 522, A44

Zapata, L.A., Schmid-Burgk, Ho, P.T.P., et al., 2009, ApJ, 704, L45 